\title{
Формирование графеноподобного слоя SiN на поверхности (111)Si
}

\author{
(C) В.Г. Мансуров ${ }^{1}$, Ю. Г. Галицын ${ }^{1}$, Т.В. Малин ${ }^{1}$, С.А. Тийс ${ }^{1}$, Е.В. Федосенко ${ }^{1}$, \\ A.C. Кожухов ${ }^{1}$, К.C. Журавлев ${ }^{1,2}$, Ildikó Cora ${ }^{3}$, Béla Pécz ${ }^{3}$ \\ ${ }^{1}$ Институт физики полупроводников им. А.В. Ржанова Сибирского отделения Российской академии наук, \\ 630090 Новосибирск, Россия \\ ${ }^{2}$ Новосибирский государственный университет, \\ 630090 Новосибирск, Россия \\ ${ }^{3}$ Thin Film Physics Department, Institute for Technical Physics and Materials Science, \\ Centre for Energy Research, Hungarian Academy of Sciences, \\ Budapest, Hungary \\ E-mail: mansurov@isp.nsc.ru
}

(Получена 25 апреля 2018 г. Принята к печати 7 мая 2018 г.)

Исследована кинетика образования и термического разложения двумерного нитридного слоя $\mathrm{SiN}-(8 \times 8)$ на поверхности (111)Si. Структура $\operatorname{SiN}-(8 \times 8)$ является метастабильной промежуточной фазой при нитридизации кремния с образованием стабильной аморфной фазы $\mathrm{Si}_{3} \mathrm{~N}_{4}$. Исследование структуры $\mathrm{SiN}-(8 \times 8)$ методом сканирующей туннельной микроскопии показывает ее сложное строение, она состоит из адсорбционной фазы $(8 / 3 \times 8 / 3)$ с латеральным периодом $10.2 \AA$ и сотовой структуры со стороной шестиугольника $\sim 6 \AA$, который развернут относительно адсорбционной фазы на $30^{\circ}$. Измерена ширина запрещенной зоны $\mathrm{SiN}-(8 \times 8)$, она равна $\sim 2.8$ эВ, что меньше в сравнении с шириной запрещенной зоны кристаллической фазы $\beta$ - $\mathrm{Si}_{3} \mathrm{~N}_{4} 5.3$ эВ. Измерены межплоскостные расстояния в структуре $(\mathrm{AlN})_{3} /(\mathrm{SiN})_{2}$ на поверхности (111) $\mathrm{Si}$, равные $3.3 \AA$ в $\mathrm{SiN}$ и $2.86 \AA$ в $\mathrm{AlN}$. Такие межплоскостные расстояния указывают на слабое ван-дер-ваальсово взаимодействие между слоями. Предложена модель структуры $\operatorname{SiN}-(8 \times 8)$ как плоского графеноподобного слоя, удовлетворяющая как дифракционным, так и микроскопическим данным.

DOI: $10.21883 /$ FTP.2018.12.46748.27

\section{1. Введение}

Нитрид кремния $\left(\mathrm{Si}_{3} \mathrm{~N}_{4}\right)$ широко применяется в различных областях, таких как металлургия, медицина, автомобильная промышленность, в качестве высокотемпературной керамики и т.д. В электронной промышленности $\mathrm{Si}_{3} \mathrm{~N}_{4}$ используется в качестве изолирующих слоев, поскольку $\mathrm{Si}_{3} \mathrm{~N}_{4}$ - один из лучших диэлектриков, имеющий высокое электрическое сопротивление $\left(10^{16} \mathrm{OM} \cdot \mathrm{cm}\right)$ и большое напряжение пробоя $\left(10^{7} \mathrm{~B} / \mathrm{cm}\right)$ [1]. Кроме того, слои $\mathrm{Si}_{3} \mathrm{~N}_{4}$ в электронных приборах создают барьер, препятствующий диффузии нежелательных примесей. В последнее время в электронике применяются ультратонкие слои $\mathrm{Si}_{3} \mathrm{~N}_{4}$ в связи с повышением плотности транзисторов в сверхбольших интегральных схемах [2,3].

Ультратонкие высококачественные слои $\mathrm{Si}_{3} \mathrm{~N}_{4}$ используются в новейших разработках в области нанотехнологий [4-7], например, при формировании наностержней $\mathrm{A}^{\mathrm{III}}$-нитридов на кремниевой подложке $[5,6]$, при изготовлении полевых электронных приборов на основе графена, позволяющих управлять перекрытием зон в графене при приложении внешнего смещения [7], а также в качестве подложки для формирования графена и силицена без потери высокой подвижности электронов в этих уникальных материалах [8-10]. Особый интерес вызывают двумерные кристаллические слои $\mathrm{SiN}$, перспективные для формирования графеноподобных и вандер-ваальсовых кристаллов.

В 1967 г. Ван Боммель и Мейер [11] впервые обнаружили упорядоченную поверхностную структуру нитрида кремния на (111)Si. В единицах латеральной постоянной решетки поверхности $(111)$ кремния $\left(a_{(111) \mathrm{Si}}=3.84 \AA\right)$ эту структуру можно обозначить как $(8 \times 8)$. Позднее Шротт и Фэйн $[12,13]$ изучили образование структуры $\mathrm{SiN}-(8 \times 8)$, они также обнаружили еще одну упорядоченную структуру, так называемую квадруплетную структуру. В работах [11-13] не было предложено моделей атомного строения структуры $\mathrm{SiN}-(8 \times 8)$, однако дифракционный рефлекс (0 11/8), наблюдаемый в дифракции медленных электронов (ДМЭ), рассматривался как фундаментальный рефлекс от новой фазы. В дальнейшем изучению структуры $\operatorname{SiN}-(8 \times 8)$, возникающей на поверхности (111) Si, уделялось большое внимание, были обнаружены и описаны ее „модификации“, такие как $(8 / 11 \times 8 / 11),(8 / 3 \times 8 / 3)$ [14-33]. Остановимся на наиболее существенных, на наш взгляд, исследованиях и моделях.

В 1984 г. авторы работ [14-16] первыми предложили модель атомного строения структуры $\mathrm{SiN}-(8 \times 8)$ [14-16]. В модели предполагалось, что она представляет собой гофрированный бислой $\mathrm{SiN}$, состоящий из нижележащей плоскости атомов $\mathrm{Si}$ и вышележащей плоскости атомов $\mathrm{N}$, с латеральной постоянной решетки $2.79 \AA$, причем $11 \times a_{\mathrm{SiN}}=8 \times a_{\mathrm{Si}}(3.84 \AA)$, где $a_{\mathrm{SiN}}=2.79 \AA, a_{\mathrm{Si}}=3.84 \AA$. Полагалось, что в этом бислое связи между атомами построены на основе $s p^{3}$-гибридизации атомных орбиталей. Для квадруплетной структуры авторы предложили планарную структуpy SiN, в которой все атомы лежат в одной плоскости и соединены $s p^{2}$-гибридизованными атомными орбиталя- 
ми. Детали предложенных моделей и устройство интерфейса с поверхностью (111)Si авторы не обсуждали.

Для объяснения структуры $(8 / 3 \times 8 / 3)$, наблюдаемой методом сканирующей туннельной микроскопии (СТМ), в работе [17] была предложена модель нитридизованного слоя на поверхности (111)Si с экзотическим составом $\mathrm{Si}_{5} \mathrm{~N}_{3}$. Авторы [18] предложили более детальную модель структуры $(8 / 3 \times 8 / 3)$ на кремнии - модель гофрированного бислоя $\mathrm{SiN}$, и при этом рассмотрели интерфейс между $\mathrm{SiN}$ и поверхностью (111)Si. Авторы сделали попытку согласовать особенности структур $(8 / 11 \times 8 / 11)$ и $(8 / 3 \times 8 / 3)$, наблюдавшихся ранее в ДМЭ и СТМ соответственно. Отметим, что в этой модели предполагается много ненасыщенных оборванных связей между слоем $\mathrm{SiN}$ и поверхностью кремния. Авторы работы [19] впервые предположили, что структура $(8 / 3 \times 8 / 3)$ формируется на основе кристаллической решетки $\beta-\mathrm{Si}_{3} \mathrm{~N}_{4}$, однако никакой детальной атомной модели этой структуры и границы $\beta-\mathrm{Si}_{3} \mathrm{~N}_{4} /(111) \mathrm{Si}$ авторы не представили.

Авторы работы [20] в 2001 г. развили более сложную модель структуры $(8 \times 8)$, которая в настоящее время широко применяется. Согласно этой модели, структуpa $\mathrm{SiN}-(8 \times 8)$ является монослоем кристаллического $\beta-\mathrm{Si}_{3} \mathrm{~N}_{4}$ толщиной $2.9 \AA$ на поверхности (111) $\mathrm{Si}$, с дополнительным слоем адатомов азота (по 9 адатомов на элементарную ячейку $(8 \times 8))$. Модель также включает атомные пары $\mathrm{Si}-\mathrm{N}$ в релаксированном слое $\beta-\mathrm{Si}_{3} \mathrm{~N}_{4}$ (3 пары на элементарную ячейку). На наш взгляд, мало вероятно, чтобы при высоких температурах $\left(>1000{ }^{\circ} \mathrm{C}\right)$, при которых формируется и хорошо наблюдается в дифракции структура $\mathrm{SiN}-(8 \times 8)$, на поверхности существовал упорядоченный адсорбированный слой атомов азота, поскольку произойдет образование молекул $\mathrm{N}_{2}$ и их десорбция. Напомним, что энергия связи (т. е. глубина потенциальной ямы) в молекуле $\mathrm{N}_{2}$ имеет весьма большую величину, 9.77 эВ, что значительно выше характерных энергий хемосорбции и поверхностных барьеров (0.1-2 эB). Существование атомных пар $\mathrm{Si}-\mathrm{N}$, предполагаемых в модели [20], также не нашло подтверждения в недавней работе [10].

В работе [20] и подобных ей, в которых за основу берется слой $\beta$ - $\mathrm{Si}_{3} \mathrm{~N}_{4}$, обычно подчеркивается, что постоянная решетки $\beta-\mathrm{Si}_{3} \mathrm{~N}_{4}(7.61 \AA)$ приблизительно в 2 раза больше, чем постоянная решетки (111)Si (3.84 $\AA$ ), что позволяет обеспечить хорошее согласование решеток $\beta-\mathrm{Si}_{3} \mathrm{~N}_{4}$ и подложки (111)Si. В некоторых работах такое совпадение постоянных решетки считается достаточным для обеспечения даже эпитаксиального роста $\beta-\mathrm{Si}_{3} \mathrm{~N}_{4}$ до толщин 20-40 $\AA$ на подложке $(111) \mathrm{Si}[21,22]$. Более того, было предложено структуру $\operatorname{SiN}-(8 \times 8)$ обозначать как $(4 \times 4)$, поскольку ее появление связывают с реконструкцией поверхности $\beta-\mathrm{Si}_{3} \mathrm{~N}_{4}$, и, следовательно, выражать ее надо не в единицах постоянной решетки кремния (111), а в единицах постоянной решетки $\beta-\mathrm{Si}_{3} \mathrm{~N}_{4}[21]$. Если принять эту модель, тогда дифракционными методами ДМЭ или ДБЭ (дифракции быстрых электронов) должен хорошо наблюдаться фундаментальный рефлекс
$\beta-\mathrm{Si}_{3} \mathrm{~N}_{4}$ ( 0 1), который соответствует дробному рефлексу $(0$ 1/2) кремния. Однако такой рефлекс при нитридизации кремния никогда не наблюдается, но хорошо видны яркие рефлексы $(0$ 3/8), (0 5/8) и (0 11/8). Нет экспериментальных подтверждений существования реконструкции $(4 \times 4)$ на поверхности монокристалла $\beta-\mathrm{Si}_{3} \mathrm{~N}_{4}$, которая могла бы быть ответственна за наблюдаемую структуру $(8 \times 8)$ [21]. При исследовании поверхности $\beta-\mathrm{Si}_{3} \mathrm{~N}_{4}$ методом высокоразрешающей просвечивающей электронной микроскопии (ПЭМВР) наблюдалась только структура $(1 \times 1)$, но не наблюдалась реконструкция поверхности $(4 \times 4)$ [23]. В расчетах из первых принципов [24,25] сообщалось о релаксации положения поверхностных атомов на идеальной поверхности (0001) $\beta-\mathrm{Si}_{3} \mathrm{~N}_{4}$, а также об образовании $\pi$-связей вместо оборванных связей на поверхности, но нет данных о реконструкции поверхности $\beta-\mathrm{Si}_{3} \mathrm{~N}_{4}$. Несмотря на указанные недостатки, именно модели структуры $\operatorname{SiN}-(8 \times 8)$, в основе которых лежит кристаллическая фаза $\beta-\mathrm{Si}_{3} \mathrm{~N}_{4}$, получили широкое распространение [19-33].

Необходимо указать, что отсутствуют термодинамические данные о фазе $(8 \times 8)$, не изучены кинетика и механизм образования структуры $\mathrm{SiN}-(8 \times 8)$ и ее модификаций $(8 / 11 \times 8 / 11),(8 / 3 \times 8 / 3)$, их области стабильности и термическое разложение. Эти данные могут дать существенную информацию о скоростях поверхностных реакций, о природе активных частиц, участвующих в образовании этих структур. Не выяснено, является ли процесс образования фазы $\mathrm{SiN}-(8 \times 8)$ термически активированным. Обычная нитридизация кремния - это термически активированный процесс, в результате которого образуется стабильная аморфная фаза $\mathrm{Si}_{3} \mathrm{~N}_{4}$. Поэтому образование промежуточной структуры $\mathrm{SiN}-(8 \times 8)$ до образования аморфной фазы $\mathrm{Si}_{3} \mathrm{~N}_{4}-$ это необычный процесс, требующий тщательного изучения. Нам известны всего несколько работ по кинетике образования структур $(8 \times 8),(8 / 11 \times 8 / 11),(8 / 3 \times 8 / 3)$ (см., например, работы $[17,32,33])$. Однако в этих работах исследованы более поздние стадии нитридизации включая стадии образования аморфного $\mathrm{Si}_{3} \mathrm{~N}_{4}$. Так, например, в недавней работе [32] кинетика исследовалась методами ДБЭ и рентгеновской фотоэлектронной спектроскопии (РФЭС). Однако экспериментальные точки на кинетической кривой показывают процесс начиная с 15-ой минуты нитридизации, хотя в картинах ДБЭ, приведенных в той же работе, видно, что образование структуры $(8 / 3 \times 8 / 3)$ происходит в течение $3-8$ с.

Таким образом, к настоящему времени имеется значительное количество экспериментального материала о структуре $\mathrm{SiN}-(8 \times 8)$, предложены атомные модели этой структуры, однако интерпретации противоречивы и ни одна из предложенных моделей не описывает весь имеющийся набор экспериментальных данных. В данной работе изучена структура $\operatorname{SiN}-(8 \times 8)$, кинетика и механизмы ее формирования и термического разложения, приводятся экспериментальные доказательства того, что при нитридизации (111)Si образуется 
метастабильная графеноподобная фаза, построенная на основе $s p^{2}$-гибридизации атомных орбиталей кремния и азота. В работе предложена новая модель структуры $\mathrm{SiN}-(8 \times 8)$.

\section{2. Эксперимент}

Эксперименты проводились в установках аммиачной молекулярно-лучевой эпитаксии СВE-32 фирмы Рибер и на сканирующем микроскопе СТМ/СТС фирмы Омикрон, установленном в сверхвысоковакуумной камере фирмы Рибер. Экспериментальные исследования in situ кинетики образования структуры $\mathrm{SiN}-(8 \times 8)$ и аморфного слоя $\mathrm{Si}_{3} \mathrm{~N}_{4}$ на поверхности (111) $\mathrm{Si}$ в потоке аммиака проводились методом ДБЭ.

Для дифракционных экспериментов использовались нелегированные подложки (111)Si диаметром 2 дюйма. Для СТМ-исследований образцы кремния размером $12 \times 3 \times 0.4$ мм вырезались из кремниевых подложек $n$-типа проводимости с сопротивлением $0.5 \mathrm{OM} \cdot \mathrm{cm}$. Отклонение поверхности от плоскости (111) не превышало $0.5^{\circ}$. Удаление защитного окисла прогревом в вакууме позволяло воспроизводимо получать атомарночистую поверхность $\mathrm{Si}$ с реконструкцией $(7 \times 7)$. В вакуумной камере образцы предварительно обезгаживались не менее 4ч при температуре $\sim 600^{\circ} \mathrm{C}$, затем поверхность кремния очищалась прогревом при $1250^{\circ} \mathrm{C}$ в течение нескольких секунд при давлении не выше $(1-2) \cdot 10^{-10}$ Торр. Образцы для СТМ-исследований прогревались прямым пропусканием тока. Калибровка температуры образца проводилась с помощью оптического пирометра. Химическое состояние поверхности кремния после нитридизации исследовалось с помощью РФЭС. Для измерения межплоскостных расстояний применялась ПЭМВР.

\section{3. Результаты и обсуждение}

B наших экспериментах при экспозиции чистой поверхности (111) Si в потоке аммиака в картинах ДБЭ наблюдается образование поверхностной структуры $(8 \times 8)$, в согласии с литературными экспериментальными данными, полученными с применением ДМЭ, ДБЭ, СТМ и других методик, описанных во Введении. На рис. 1 представлена дифракционная картина, полученная после экспозиции поверхности при температуре $T=1050^{\circ} \mathrm{C}$ в течение $6 \mathrm{c}$ в потоке аммиака $10 \mathrm{~cm}^{3} /$ мин в стандартных условиях. Наряду с фундаментальными рефлексами кремния $(00),(01)$ и $(0 \overline{1})$ отчетливо наблюдаются следующие яркие рефлексы нитридной фазы: (0 3/8), (0 5/8), (0 6/8), (0 11/8); более слабо проявляются рефлексы $(0$ 1/8), (0 2/8) и $(07 / 8)$, однако не наблюдается рефлекс $(04 / 8)$. Наряду с изучением статической структуры образующегося нитридного слоя $(8 \times 8)$ важную информацию дает исследование кинетики процесса ее образования дифракционным методом.

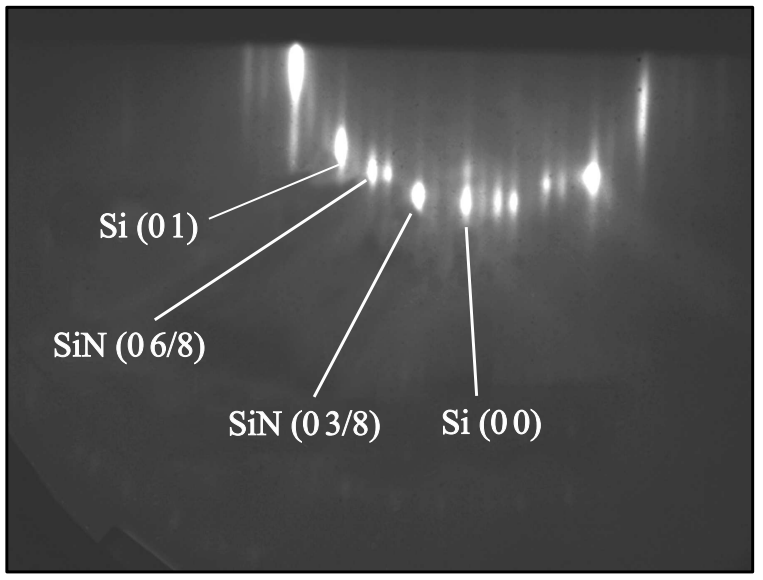

Рис. 1. Дифракционная картины $\mathrm{SiN}-(8 \times 8)$ (0001) SiN/ (111)Si вблизи азимутального направления [1 110$]$.

Кинетика образования нитридной пленки при нитридизации уже исследовалась нами ранее методом ДБЭ в работе [33]. Было обнаружено, что дробные рефлексы структуры $\mathrm{SiN}-(8 \times 8)$ появляются в картинах ДБЭ в течение нескольких секунд $(5-7 \mathrm{c})$. При дальнейшей нитридизации в тех же условиях дифракционная картина в течение нескольких минут гаснет, что объясняется формированием аморфного слоя $\mathrm{Si}_{3} \mathrm{~N}_{4}$. Было показано, что в образовании структуры $\mathrm{SiN}-(8 \times 8)$ основную роль играют подвижные адатомы кремния, находящиеся в равновесии с поверхностью кремния при заданной температуре. Степень заполнения поверхности фазой $\mathrm{SiN}-(8 \times 8)$ увеличивается с температурой, которая и определяется равновесной концентрацией подвижных адатомов кремния. Была также определена теплота образования подвижных адатомов $\mathrm{Si} 1.7$ э. Так как скорость образования структуры $\mathrm{SiN}-(8 \times 8)$ высокая, равновесная концентрация подвижных адатомов кремния не успевает восстановиться.

В данной работе мы изучили стабильность фазы $\mathrm{SiN}-(8 \times 8)$ при различных температурах. Прежде всего обратим внимание, что образование структуры $\mathrm{SiN}-(8 \times 8)$ при экспозиции чистой поверхности кремния $(1 \times 1)$ в потоке аммиака при высокой температуре происходит в результате фазового перехода, когда на поверхности накопится достаточное количество связей $\mathrm{Si}-\mathrm{N}$, как было показано в нашей предыдущей работе [33]. Поскольку после быстрого процесса образования упорядоченной структуры $\mathrm{SiN}-(8 \times 8)$ в потоке аммиака происходит медленное образование более стабильной аморфной фазы $\mathrm{Si}_{3} \mathrm{~N}_{4}$ с энергией активации 2.4 эВ [33], можно заключить, что фаза $\mathrm{SiN}-(8 \times 8)$ является метастабильной. Этот экспериментальный факт не согласуется с предполагаемым эпитаксиальным ростом кристалла $\beta-\mathrm{Si}_{3} \mathrm{~N}_{4}$ на поверхности кремния при нитридизации $[21,22]$. Для проверки метастабильности фазы $\mathrm{SiN}-(8 \times 8)$ мы изучили кинетику 


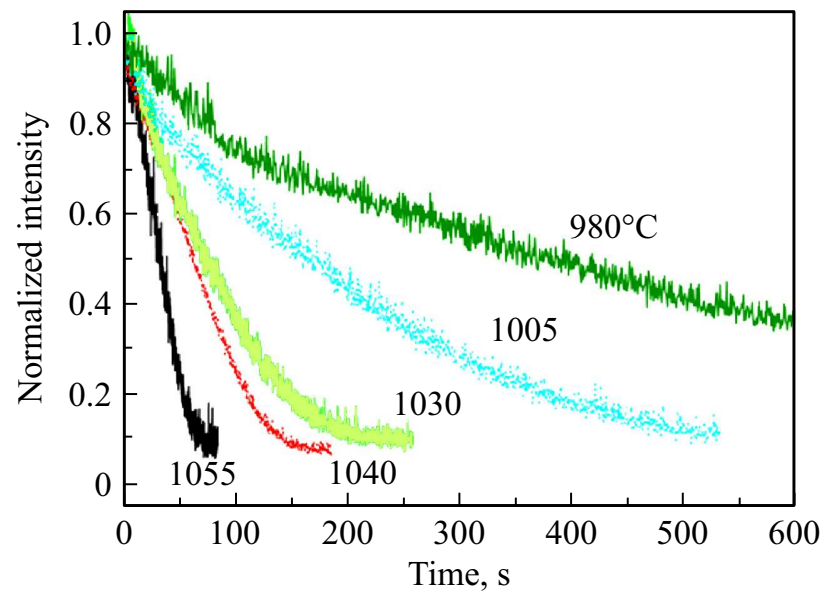

Рис. 2. Эволюция интенсивности рефлекса (0 3/8) в процессе термического разложения фазы $\operatorname{SiN}-(8 \times 8)$ при различных температурах.

ее разложения в вакууме. В этих экспериментах наблюдалось восстановление картины $(1 \times 1)$ чистой поверхности кремния при прогреве образцов выше $980^{\circ} \mathrm{C}$. На рис. 2 представлены кинетические кривые термического разложения фазы $\operatorname{SiN}-(8 \times 8)$. Кинетические кривые аппроксимировались спадающей экспонентой интенсивности $I(t)=I_{0} \exp [-k(T) / t]$, где $t-$ время, $k=k_{0} \exp \left(-E_{a} / k_{\mathrm{B}} T\right)-$ кинетическая константа разложения фазы $\mathrm{SiN}-(8 \times 8), T-$ температура поверхности, $k_{\mathrm{B}}$ - постоянна Больцмана. Определены энергия активации термического разложения $\operatorname{SiN}-(8 \times 8), E_{a}=4.03$ эВ, и предэкспоненциальный множитель $k_{0}=2.4 \cdot 10^{13} 1 / \mathrm{c}$. Энергия активации оказалась близка к известной энергии связи $\mathrm{Si}-\mathrm{N} 4.5$ эВ [6]. Поскольку энергия активации разложения не может быть меньше теплоты образования фазы $\mathrm{SiN}-(8 \times 8)\left(E_{a} \geq \Delta H\right)$, следовательно, $\Delta H \leq 4$ эВ. Отметим, что теплота образования $\beta-\mathrm{Si}_{3} \mathrm{~N}_{4}$ составляет $\sim 8$ эВ [34], т.е. значительно больше оцененной здесь теплоты образования фазы $\operatorname{SiN}-(8 \times 8)$, что подтверждает ее метастабильность. Интересно, что энергия активации поверхностного процесса термического разложения $\mathrm{Si}_{3} \mathrm{~N}_{4}, \sim 4$ эВ [34], совпадает с измеренной нами энергией активации разложения $\operatorname{SiN}-(8 \times 8)$. Совпадение энергий активации свидетельствует о том, что в обоих случаях лимитирующей стадией процесса является разрыв связи $\mathrm{Si}-\mathrm{N}$. Однако предэкспоненциальный множитель термораспада фазы $\mathrm{SiN}-(8 \times 8)$ на 6 порядков выше, чем для поверхностного разложения $\mathrm{Si}_{3} \mathrm{~N}_{4}$, равный $3.9 \cdot 10^{7} 1 / \mathrm{c}$ [34], следовательно, термораспад фазы $\mathrm{SiN}-(8 \times 8)$ не совпадает с процессом поверхностного разложения $\mathrm{Si}_{3} \mathrm{~N}_{4}$. Отметим также, что измеренный нами предэкспоненциальный множител имеет нормальное значение $\sim 10^{13} 1 / \mathrm{c}$, что предполагает простой механизм разложения $\mathrm{SiN}-(8 \times 8)$. Происходит разрыв связей $\mathrm{Si}-\mathrm{N}$ и рекомбинация атомов азота с образованием молекулы $\mathrm{N}_{2}$.
На нитридизованной поверхности (111)Si со структурой $\mathrm{SiN}-(8 \times 8)$ методом РФЭС измерялись химические сдвиги остовных уровней в атомах кремния $(\mathrm{Si} 2 p)$ и азота $(\mathrm{N} 1 s)$. На рис. 3 показан РФЭС-спектр структуры $\mathrm{SiN}-(8 \times 8)$, полученной при нитридизации в течение $6 \mathrm{c}$ при температуре $1050^{\circ} \mathrm{C}$. В спектре пик $\mathrm{Si}^{0}$ соответствует объемному кремнию, положение этого фотоэлектронного пика на шкале энергий связи имеет значение 99 эВ; кроме того, отчетливо наблюдается плечо $\left(\mathrm{Si}^{3+}\right)$ с положением пика около 101.3 эВ. Это значение соответствует атомам кремния, связанным с 3 атомами азота. Известно, что энергия связи $\mathrm{Si} 2 p$ в $\mathrm{Si}_{3} \mathrm{~N}_{4}$ (где кремний связан с четырьмя атомами азота) имеет значение в диапазоне 101.7-102 эВ, т.е. больше, чем в нашем случае [26,35-37]. Мы оценили также состав $\operatorname{SiN}-(8 \times 8)$, который оказался близок к составу $\mathrm{SiN}$ (т.е. $[\mathrm{Si}]:[\mathrm{N}] \approx 1: 1)$ с небольшим обогащением по кремнию, т.е. состав $\mathrm{SiN}-(8 \times 8)$ не соответствует составу $[\mathrm{Si}]:[\mathrm{N}]=0.75$, как должно быть в слое $\mathrm{Si}_{3} \mathrm{~N}_{4}$.

Атомное строение структуры $\operatorname{SiN}-(8 \times 8)$ в реальном пространстве мы исследовали методами СТМ и ПЭМВР.

Изображение структуры $\mathrm{SiN}-(8 \times 8)$, измеренное методом СТМ при напряжении на образце $V_{s}=-3 \mathrm{~B}$, т.е. в заполненных состояниях, показано на рис. 4. Отчетливо проявляется периодическая структура $(8 / 3 \times 8 / 3)$ с расстоянием между ближайшими соседними светлыми „шарами“ $\sim 10.2 \AA$, в согласии с литературными данными $[10,17,18,21,22]$. Однако мы обнаружили дополнительную сотовую структуру, шестиугольные ячейки которой развернуты на $30^{\circ}$ относительно элементарных ячеек структуры $(8 / 3 \times 8 / 3)$. Длина стороны ячейки сотовой структуры составляет $\sim 6 \AA$, расстояние между центрами соседних ячеек $10.2 \AA$, т.е. такое же, как и сторона элементарной ячейки $(8 / 3 \times 8 / 3)$.

Поскольку наблюдаемые „шары“ структуры $(8 / 3 \times 8 / 3)$ светлее ячеек сотовой структуры, можно предположить, что „шары“ лежат наверху сотовой структуры. Мы предполагаем, что светлые „шары“ яв-

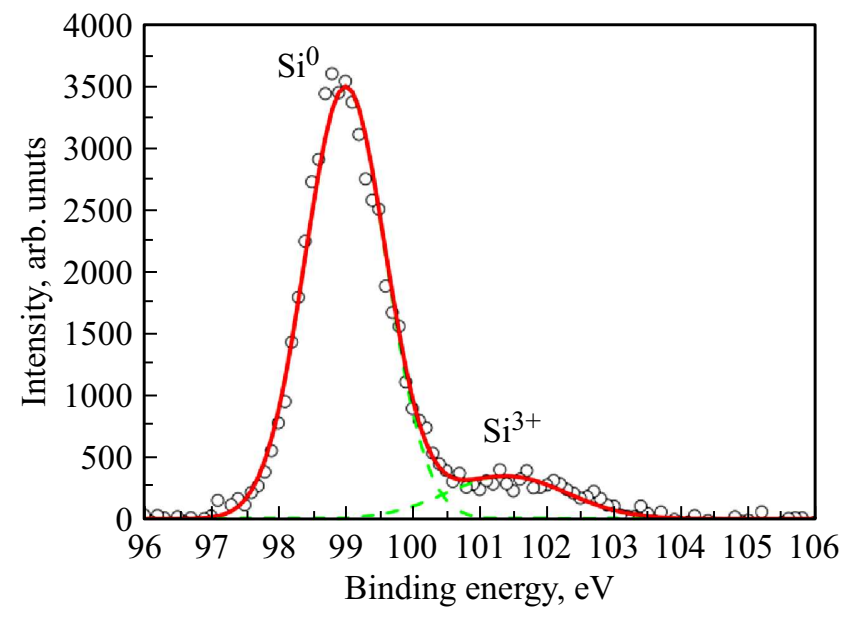

Рис. 3. РФЭС-спектр $\mathrm{Si} 2 p$ нитридизованной поверхности со структурой $\mathrm{SiN}-(8 \times 8)$. 


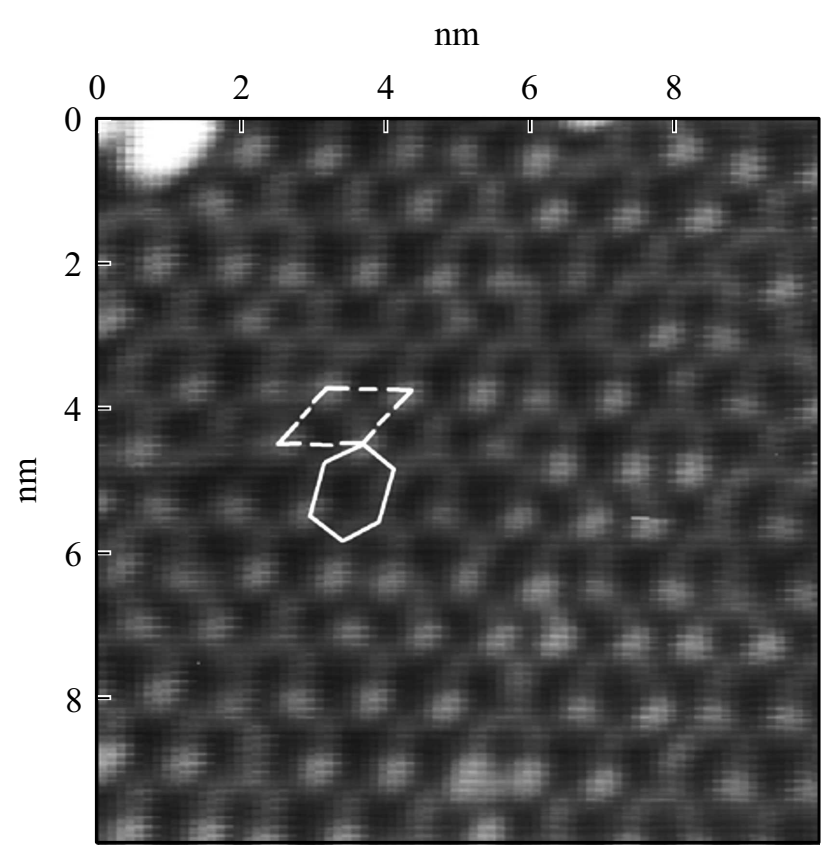

Рис. 4. СТМ-картина структуры $\operatorname{SiN}-(8 \times 8)$, измерения при $V_{s}=-3$ эВ. Сплошной линией показана ячейка сотовой структуры, сторона гексагона $\sim 6 \AA$. Штриховой линией показана элементарная ячейка фазы адсорбированных атомов кремния с периодом $10.2 \AA$.

ляются адсорбированными атомами кремния на сотовой структуре. Эти адатомы кремния подвижны, поскольку на рисунке видны как вакансии, т.е. узлы, не занятые адатомами кремния, так и адатомы, расположенные на расстояниях $6 \AA$, т. е. в ближайших узлах ячейки сотовой структуры.

Мы измерили также вольт-амперные характеристики туннельного тока в СТМ и обнаружили увеличение „бестокового зазора“ до $\sim 2.8$ эВ между заполненными и пустыми состояниями по сравнению с чистой поверх- ностью (111) $\mathrm{Si}$, где он равен 1.1 эВ. Обратим внимание, что эта величина заметно меньше ширины запрещенной зоны $\mathrm{Si}_{3} \mathrm{~N}_{4}(5-5.3$ эВ).

На рис. 5 представлено ПЭМВР-изображение кристаллической структуры, состоящей из двух монослоев $\mathrm{SiN}$ и трех монослоев AlN, эпитаксиально выращенных на поверхности (111)Si. Видно, что расстояние между поверхностным слоем кремния и слоем $\mathrm{SiN}$, а также между слоями $\mathrm{SiN}$ составляет $3.3 \AA$, что заметно больше толщины монослоя в кристалле $\beta-\mathrm{Si}_{3} \mathrm{~N}_{4}(2.9 \AA)$ и больше межплоскостных расстояний в $\mathrm{Si}(3.13 \AA)$. Следовательно, взаимодействие между поверхностью кремния и слоем SiN является более слабым, чем ковалентное химическое взаимодействие, и носит ван-дер-ваальсов характер. Обратим внимание, что расстояние между слоями $\mathrm{SiN}$ и $\mathrm{AlN}$ составляет $3.22 \AA$, а расстояние между слоями $\mathrm{AlN}$ составляет $2.86 \AA$, что также заметно больше, чем толщина монослоя в кристалле $\mathrm{AlN}(2.49 \AA)$. Ранее в работе [38] для формирования графеноподобного слоя AlN нами уже использовался нитридизованный слой кремния со структурой $\mathrm{SiN}-(8 \times 8)$ в качестве подложки. Если бы слой $\mathrm{SiN}-(8 \times 8)$ имел оборванные ненасышенные связи, тогда невозможно было бы получить графеноподобные слои AlN.

На основе анализа литературных данных и новых данных, полученных в данной работе, мы предлагаем новую модель структуры $\operatorname{SiN}-(8 \times 8)$, основанную на $s p^{2}$-гибридизации атомных орбиталей кремния и азота. Известно, что атом азота в кристалле $\beta-\mathrm{Si}_{3} \mathrm{~N}_{4}$ связан с 3 атомами кремния в плоской конфигурации $[39,40]$, угол между связями близок к $120^{\circ}$, т.е. атомные орбитали азота находятся в $s p^{2}$-гибридизации. С другой стороны, атомные орбитали кремния также могут находиться в $s p^{2}$-гибридирзации, как, например, в силицене [41-43] или как атомы кремния, связанные с азотом на поверхности $\beta-\mathrm{Si}_{3} \mathrm{~N}_{4}[24,25]$. Ранее мы уже подчеркивали, что образование метастабильной фазы $\mathrm{SiN}$ происходит с участием подвижных адатомов кремния.

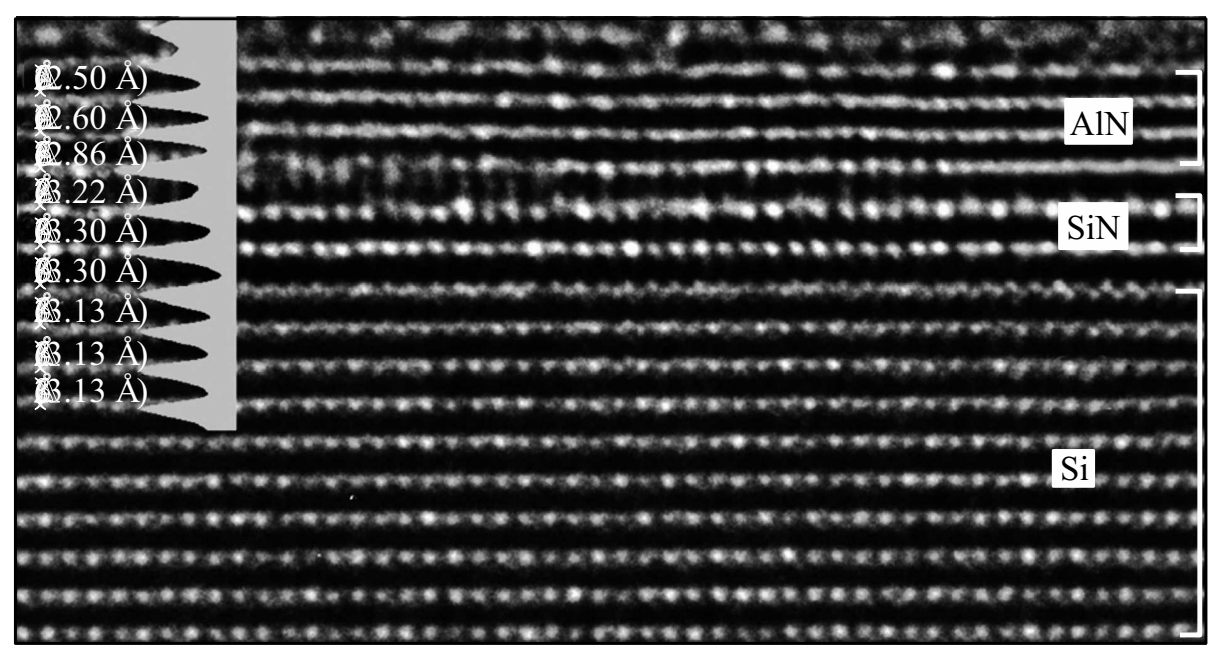

Рис. 5. Картина ПЭМВР слоев графеноподобного $\mathrm{SiN}$ на поверхности (111) Si. Межплоскостные расстояния в $\mathrm{SiN} 3.3 \AA$. 


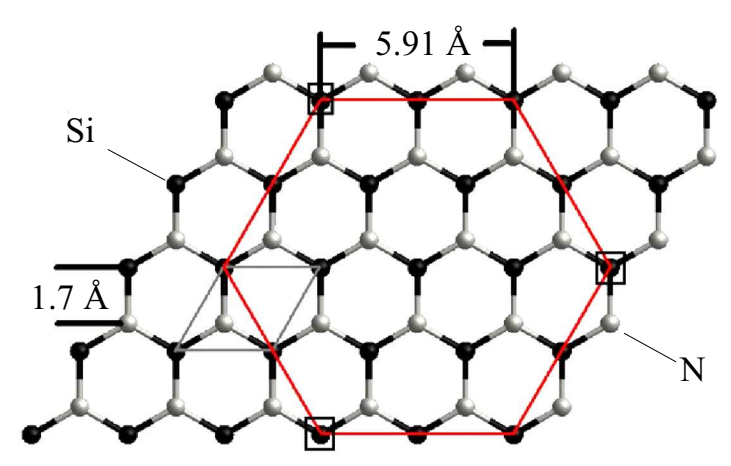

Рис. 6. Модель графеноподобного слоя SiN.

Другими словами, в образовании этой фазы не участвуют „неподвижные“ поверхностные атомы, находящиеся в узлах кристалла кремния. Этот процесс аналогичен образованию силицена на поверхности (111) серебра, где подвижные адатомы кремния образуют различные промежуточные силиценовые фазы [44], прежде чем образуется аморфный или кристаллический кремний при дальнейшем нанесении кремния. Подобным образом, как было показано нами [33], метастабильная фаза $\mathrm{SiN}\left(s p^{2}\right)$ является промежуточной, прежде чем образуется стабильная фаза $\mathrm{Si}_{3} \mathrm{~N}_{4}$, в которой атомные орбитали кремния преобразуются из $s p^{2}$ - в $s p^{3}$-гибридизованные орбитали.

Таким образом, данные ВРЭМ показывают, что фаза SiN имеет плоскую графеноподобную структуру, слои которой слабо взаимодействуют с поверхностью кремния. Данные РФЭС также подтверждают, что атом кремния связан с 3 атомами азота и, следовательно, имеет $s p^{2}$-гибридизацию.

Модель графеноподобного слоя $\mathrm{SiN}$ представлена на рис. 6. Длина связи $\mathrm{Si}-\mathrm{N}$ в кристалле $\beta-\mathrm{Si}_{3} \mathrm{~N}_{4}$ лежит в диапазоне $1.704-1.767 \AA$ [39]. Учитывая, что в плоских гексагональных структурах при добавлении $\pi$-связи происходит укорочение связи, в модели мы выбрали значение длины связи $\mathrm{Si}-\mathrm{N} \quad 1.707 \AA$. Это значение определяет длину стороны элементарной ячейки графеноподобной структуры $\mathrm{SiN}-2.956 \AA=\sqrt{3} \cdot 1.707 \AA$, а также определяет сторону шестиугольника со стороной $5.91 \AA=2 \sqrt{3} \cdot 1.707 \AA$, который наблюдается в СТМ-изображении как ячейка сотовой структуры размером $\sim 6 \AA$ (см. рис. 4). Выбранная длина связи также хорошо согласуется с периодом $10.2 \AA=\sqrt{3} \cdot 5.91 \AA$ адсорбированной фазы $(8 / 3 \times 8 / 3)$. На рисунке квадратами очерчены положения атомов кремния в адсорбированной фазе $(8 / 3 \times 8 / 3)$. Легко увидеть, что предложенная модель согласуется и с данными ДБЭ: например, яркий рефлекс $(0-3 / 8)$ на рис. 1 соответствует вектору обратной решетки адсорбированной фазы $1 / 10.2 \AA^{-1}$, рефлекс $(0-6 / 8)$ соответствует вектору обратной решетки $1 / 5.1 \AA^{-1}$, где $5.1 \AA=\sqrt{3} \cdot 2.956 \AA$.

\section{4. Заключение}

Таким образом, в работе исследованы кинетика образования и термического разложения двумерного нитридного слоя на поверхности (111)Si. Показана метастабильность фазы SiN- $(8 \times 8)$. Проведены СТМ-исследования атомной структуры $\mathrm{SiN}-(8 \times 8)$, которые показали наличие двух фаз: адсорбционной фазы $(8 / 3 \times 8 / 3)$ с периодом $10.2 \AA$, и фазы с сотовой структурой и стороной ячеки $\sim 6 \AA$, повернутой относительно адсорбционной на $30^{\circ}$. Измерена ширина запрещенной зоны $\mathrm{SiN}-(8 \times 8)-2.8$ эВ, что меньше в сравнении с запрещенной кристаллической фазы $\beta-\mathrm{Si}_{3} \mathrm{~N}_{4}(5.3$ эB). Экспериментально измерено межплоскостное расстояние между слоями $\mathrm{SiN}$ в ван-дер-ваальсовом кристалле - $3.3 \AA$. Предложена новая графеноподобная модель структуры $\mathrm{SiN}-(8 \times 8)$, которая согласуется с экспериментальными данными как дифракции, так и микроскопии. Полученные результаты будут полезны технологам для оптимизации начальных этапов эпитаксиального роста $\mathrm{A}^{\mathrm{III}}$-нитридов $(\mathrm{GaN}, \mathrm{AlN}, \mathrm{InN})$ на кремниевой подложке, а также при создании и исследовании новых графеноподобных материалов.

Работа выполнена при поддержке Российского фонда фундаментальных исследований (гранты РФФИ № 17-02-00947, 18-52-00008).

\section{Список литературы}

[1] Handbook of Semiconductor Manufacturing Technology, ed. by R. Doering, Y. Nishi (Cleveland, CRC, 2007).

[2] T.P. Ma. IEEE Trans. Electron Dev., 45, 680 (1998).

[3] Y. Yeo, Q. Lu, P. Ranade, H. Takeuchi, K.J. Yang, I. Polishchuk, T. King, Ch. Hu, S.C. Song, H.F. Luan, D. Kwong. IEEE Electron Dev. Lett., 22 (5), 227 (2001).

[4] W. Guo, M. Zhang, A. Banerjee, P. Bhattacharya. Nano Lett., 10, 3355 (2010).

[5] T.-W. Yeh, Y.-T. Lin, L.S. Stewart, P.D. Dapkus, R. Sarkissian, J.D. O’Brien, B. Ahn, S.R. Nutt. Nano Lett., 12, 3257 (2012).

[6] S. Shetty, M. Kesaria, J. Ghatak, S.M. Shivaprasad. Cryst. Growth Des., 13, 2407 (2013).

[7] W. Zhu, D. Neumayer, V. Perebeinos, P. Avouris. Nano Lett., 10, 3572 (2010).

[8] M. Yang, C. Zhang, Sh. Wang, Y. Feng, Ariando. AIP Advances, 1, 032111 (2011).

[9] F. Filippone. J. Phys. Condens. Matter, 26, 395009 (2014).

[10] R. Flammini, P. Allegrini, F. Wiame, R. Belkhou, F. Ronci, S. Colonna, D.M. Trucchi, F. Filippone, S.K. Mahatha, P.M. Sheverdyaeva, P. Moras. Phys. Rev. B, 91, 075303 (2015).

[11] A.J. van Bommel, F. Meyer. Surf. Sci., 8, 381 (1967).

[12] A.G. Schrott, J.S.C. Fain. Surf. Sci., 111, 39 (1981).

[13] A.G. Schrott, J.S.C. Fain. Surf. Sci., 123, 204 (1982).

[14] M. Nishijima, H. Kobayashi, K. Edamoto, M. Onchi. Surf. Sci., 137, 473 (1984).

[15] M. Nishijima, K. Edamoto, Y. Kubota, H. Kobayashi, M. Onchi. Surf. Sci., 158, 422 (1985). 
[16] K. Edamoto, S. Tanaka, M. Onchi, M. Nishijima. Surf. Sci., 167, 285 (1986).

[17] E. Bauer, Y. Wei, T. Muller, A. Pavlovska, I.S.T. Tsong. Phys. Rev. B, 51, 17891 (1995).

[18] Y. Morita, H. Tokumoto. Surf. Sci., 443, L1037 (1999).

[19] B. Rottger, R. Kliese, H. Neddermeyer. J. Vac. Sci. Technol. B, 14, 1051 (1996).

[20] H. Ahn, C.-L. Wu, S. Gwo, C.M. Wei, Y.C. Chou. Phys. Rev. Lett., 86, 2818 (2001).

[21] X. Wang, G. Zhai, J. Yang, N. Cue. Phys. Rev. B, 60, R2146 (1999).

[22] X.-S. Wang, G. Zhai, J. Yang, L. Wang, Y. Hu, Z. Li, J.C. Tang, X. Wang, K.K. Fung, N. Cue. Surf. Sci., 494 (2), 83 (2001).

[23] A. Ziegler, C. Kisielowski, R.O. Ritchie. Acta Materialia, 50, 565 (2002).

[24] A.A. Bagatur'yants, K.P. Novoselov, A.A. Safonov, J.V. Cole, M. Stoker, A.A. Korkin. Surf. Sci., 486, 213 (2001).

[25] V.M. Bermudez. Surf. Sci., 579, 11 (2005).

[26] J.W. Kim, H.W. Yeom. Phys. Rev. B, 67, 035304 (2003).

[27] G.L. Zhao, M.E. Bachlechner. Phys. Rev. B, 58, 1887 (1998).

[28] J.S. Ha, K.-H. Park, W.S. Yun, E.-H. Lee, S.-J. Park. J. Vac. Sci. Technol. B, 15, 1893 (1997).

[29] J.S. Ha, K.-H. Park, W.S. Yun, E.-H. Lee, S.-J. Park. Appl. Phys. A, 66, S495 (1998).

[30] C.-L. Wu, J.-L. Hsieh, H.-D. Hsueh, S. Gwo. Phys. Rev. B, 65, 045309 (2002).

[31] M. Yang, R.Q. Wu, W.S. Deng, L. Shen, Z.D. Sha, Y.Q. Cai, Y.P. Feng, S.J. Wang. J. Appl. Phys., 105, 024108 (2009).

[32] S. Shetty, S.M. Shivaprasad. J. Appl. Phys., 119, 055306 (2016).

[33] V.G. Mansurov, T.V. Malin, Yu.G. Galitsyn, A.A. Shklyaev, K.S. Zhuravlev. J. Cryst. Growth, 441, 12 (2016).

[34] H.D. Batha, E.D. Whitney. J. American Ceramic Soc., 56 (7), 365 (1973).

[35] L. Kubler, J.L. Bischoff, D. Bolmont. Phys. Rev. B, 38, 13113 (1988).

[36] D. Bolmont, J.L. Bischoff, F. Lutz, L. Kubler. Surf. Sci., 269/270, 924 (1992).

[37] H. Lee, Ch. Kuo, H. Shiu, Ch. Chen, Sh. Gwo. Appl. Phys. Lett., 95, 222104 (2009).

[38] V. Mansurov, T. Malin, Yu. Galitsyn, K. Zhuravlev. J. Cryst. Growth, 428, 93 (2015).

[39] R. Grun. Acta Cryst. B, 35, 800 (1979).

[40] H.M. Jennings, J.O. Edwards, M.H. Richman. Inorg. Chimica Acta, 20, 167 (1976).

[41] P. De Padova, C. Quaresima, B. Olivieri, P. Perfetti, Guy Le Lay. Appl. Phys. Lett., 98, 081909 (2011).

[42] B. Aufray, A. Kara, S. Vizzini, H. Oughaddou, Ch. Léandri, B. Ealet, Guy Le Lay. Appl. Phys. Lett., 96, 183102 (2010).

[43] M. Houssa, G. Pourtois, V.V. Afanas'ev, A. Stesmans. Appl. Phys. Lett., 97, 112106 (2010).

[44] B. Feng, Z. Ding, Sh. Meng, Y. Yao, X. He, P. Cheng, L. Chen, K. Wu. Nano Lett., 12, 3507 (2012).

\section{Graphene-like SiN layer formation on (111)Si surface}

V.G. Mansurov ${ }^{1}$, Yu.G. Galitsyn ${ }^{1}$, T.V. Malin ${ }^{1}$, S.A. Teys ${ }^{1}$, E.V. Fedosenko ${ }^{1}$, A.S. Kozhukhov' ${ }^{1}$, K.S. Zhurevlev ${ }^{1,2}$, Ildikó Cora ${ }^{3}$, Béla Pécz ${ }^{3}$

${ }^{1}$ Rzhanov Institute of Semiconductors Physics, Siberian Branch of Russian Academy of Sciences, 630090 Novosibirsk, Russia

${ }^{2}$ Novosibirsk State University, 630090 Novosibirsk, Russia

${ }^{3}$ Thin Film Physics Department, Institute for Technical Physics and Materials,

Science Centre for Energy Research, Hungarian Academy of Sciences,

Budapest, Hungary

Abstract The formation and thermal decomposition kinetics of a $\mathrm{SiN}-(8 \times 8)$ structure on the $(111) \mathrm{Si}$ surface was studied. A twodimensional nitride layer $\operatorname{SiN}-(8 \times 8)$ is a metastable intermediate phase appearing before formation of stable amorphous $\mathrm{Si}_{3} \mathrm{~N}_{4}$ phase. Investigations of the $(8 \times 8)$ structure by scanning tunnel microscopy method show that the $\operatorname{SiN}-(8 \times 8)$ consists of two phases: adsorption phase $(8 / 3 \times 8 / 3)$ with a lateral period of $10.2 \AA$, and honeycomb structure with a hexagon side of $\sim 6 \AA$, which is rotated by $30^{\circ}$ relative to the adsorption phase. The band gap for the $\mathrm{SiN}-(8 \times 8)$ of $2.8 \mathrm{eV}$ was measured, which is smaller than the band gap for the $\beta-\mathrm{Si}_{3} \mathrm{~N}_{4}$ phase of $5.3 \mathrm{eV}$. The interplanar spacings in the structure $(\mathrm{AlN})_{3} /(\mathrm{SiN})_{2}$ on $\mathrm{Si}$ was measured by HRTEM, being $3.3 \AA$ for the SiN layers and $2.86 \AA$ for the AlN layers. These interplanar spacings denote a weak Van der Waals interaction between the layers. A model of $\operatorname{SiN}-(8 \times 8)$ structure is proposed as a flat graphene-like layer that is in agreement both diffraction and microscopic data.

Редактор Л.В. Шаронова 